\title{
Ovarian Cancer Stem Cells
}

\author{
SHARMILA A. BAPAT
}

The hypothesis that cancer could be a stem cell disease actually existed for several years before definitive 'proof-of-concept' experiments unequivocally established their involvement in initiating the tumourigenic state. Over the last decade, the combination of fluorescence-activated cell sorting with functional read-outs e.g. in vitro colony forming units in colony initiating assays, and competitive repopulating assays in animal models has enabled the prospective purification and enrichment of cancer stem cells (CSCs) in leukemia and also in certain solid tumours. However, a major limitation for research in the field of stem cells and CSCs has been the lack of research resources in the form of in vitro models for these studies.

Earlier work in our lab provided the first evidence of the involvement of stem/progenitor cells in human ovarian tumours through the establishment of a novel $\boldsymbol{O} \mathbf{V}-\boldsymbol{C S C}$ (Ovarian Cancer Stem/Progenitor Cell) model system. Briefly, this system consists of 19 spontaneously immortalized single cell clones isolated from a single multi-layererd spheroid from malignant ovarian ascites. All these clones express several stem cell markers. One of these clones was found to be tumourigenic at the time of isolation, while another clone acquired tumourigenicity after a few passages in vitro, thereby being representative of tumour progression from a pre-transformed to transformed state. The system provides an apt tool to study CSCs and identify disruption of the normal stem cell regulation during cancer. One such application of this system was the identification of a novel mechanism of mediation of long-term angiogenesis employed by CSCs towards ensuring tumour survival. This involves the recruitment of endothelial stem cells by CSCs that have the potential to give rise to an entire hierarchy of cells thereby generating the necessary critical cellular mass required for the development of tumour vasculature. 